\title{
APPROXIMATION THEOREMS AND FIXED POINT THEOREMS IN CONES
}

\author{
TZU-CHU LIN
}

(Communicated by Paul S. Muhly)

\begin{abstract}
In this paper, we investigate the validity of an interesting theorem of Fan [3, Theorem 2] in cones. We prove that it is true for a continuous condensing map defined on a closed ball in cones. A more interesting case is that we prove that it is true on an annulus if suitable inner boundary conditions are posed. As applications of our theorems, some new fixed point theorems in the norm form are derived.
\end{abstract}

In [3], Fan proved a very interesting theorem [3, Theorem 1]; its normed space version [3, Theorem 2] is stated as follows:

Let $K$ be a nonempty compact convex set in a normed linear space $X$. For any continuous map from $K$ into $X$, there exists a point $u$ in $K$ such that

$$
\|u-f(u)\|=d(f(u), K) \text {. }
$$

Various aspects of this theorem have been studied by Fan [4], Lin [10, 11], Lin and Yen [12], Reich [16], and Sehgal and Singh [18].

On the other hand, fixed point theorems in cones are very important in applications (e.g. the survey article by Amann [1]), and have been investigated by many authors (e.g., Nussbaum [14], Fitzpatrick and Petryshyn [5], Krasnoselski [8], Các and Gatica [2], and others).

In this paper, we will investigate the validity of the above theorem for a continuous condensing map defined on a closed ball and an annulus in cones. As applications of our theorems, new fixed point theorems are derived. And we also generalize some recent results of $\mathrm{Li}$ [9] for the case of continuous condensing maps.

Now we state some definitions and notations. Let $X$ be a (real) Banach space. A nonempty subset $P$ of $X$ is called a cone if $P$ is closed, and whenever $x, y \in P$ and $\lambda, \beta \in \mathbf{R}, \lambda \geq 0, \beta \geq 0$, then $\lambda x+\beta y \in P$. We do not insist that $P \cap(-P)=\{0\}$ at this moment; we will make this requirement for cones later on. Set

$$
\begin{aligned}
& P_{r}=\{x \in P \mid\|x\|<r\}, \quad \partial P_{r}=\{x \in P \mid\|x\|=r\}, \\
& P_{R}=\{x \in P \mid\|x\|<R\}, \quad \partial P_{R}=\{x \in P \mid\|x\|=R\}, \\
& P_{r, R}=\{x \in P \mid r<\|x\|<R\}, \quad \overline{P_{r, R}}=\{x \in P \mid r \leq\|x\| \leq R\} . \\
& B(0, R)=\{x \in X \mid\|x\|<R\}, \quad 0<r<R .
\end{aligned}
$$

Received by the editors October 31,1986 . This paper was presented at the American Mathematical Society's 834th meeting held at New Jersey Institute of Technology, Newark, New Jersey, April 25-26, 1987.

1980 Mathematics Subject Classification (1985 Revision). Primary 47H07, 47H10; Secondary 41A50, 54H25. 
Let $S$ be a subset of $X$ and $x \in \bar{S}$; we denote $I_{S}(x)$ by $\{x+c(z-x) \mid$ for some $z \in S$, some $c>0\}$, which is an inward set originally studied by Halpern in [7]. We note that $S \subset I_{S}(x)$. The definitions of condensing and $k$-set-contraction maps can be found in [13] or [15].

Now we state and prove our theorems.

THEOREM 1. If $f$ is a continuous condensing map from $\overline{P_{R}}$ into $P$, then there exists a point $u$ in $\overline{P_{R}}$ such that $\|u-f(u)\|=d\left(f(u), \overline{P_{R}}\right)$.

Proof. Define $h: X \rightarrow \overline{B(0 ; R)}$ by

$$
h(x)= \begin{cases}x & \text { if }\|x\| \leq R, \\ R x /\|x\| & \text { if }\|x\| \geq R .\end{cases}
$$

From [13, Corollary 1] $h$ is a continuous 1-set-contraction. Let $F(x)=h(f(x))$. Since $P$ is a cone, we have $\overline{P_{R}}=\overline{B(0 ; R)} \cap P$ and $h: P \rightarrow \overline{B(0 ; R)} \cap P$. Therefore, $F: \overline{P_{R}} \rightarrow \overline{P_{R}}$. It is clear that $F$ is a continuous condensing map. By [6] or [17], there exists $u$ in $\overline{P_{R}}$ such that $F(u)=u$.

For this $u$, we have

$$
\begin{aligned}
\|u-f(u)\| & =\|h(f(u))-f(u)\| \\
& = \begin{cases}\|f(u)-f(u)\|=0 & \text { if }\|f(u)\| \leq R, \\
\|R f(u) /\| f(u)\|-f(u)\|=\|f(u)\|-R & \text { if }\|f(u)\| \geq R .\end{cases}
\end{aligned}
$$

For each $x \in \overline{P_{R}}$, we have

$$
\|f(u)\|-R \leq\|f(u)\|-\|x\| \leq\|f(u)-x\| .
$$

Hence $\|u-f(u)\|=\operatorname{Min}_{x \in \overline{P_{R}}}\|x-f(u)\|=d\left(f(u), \overline{P_{R}}\right)$.

THEOREM 2. If $f$ is a continuous condensing map from $\overline{P_{R}}$ into $P$, and $f$ satisfies either one of the conditions:

(i) if for each $x \in \overline{P_{R}}$ with $x \neq f(x)$, there exists $y$ in $P$ and $y$ in $I_{\overline{P_{R}}}(x)=$ $\left\{x+c(z-x) \mid\right.$ for some $z \in \overline{P_{R}}$, some $\left.c>0\right\}$ such that $\|y-f(x)\|<\|x-f(x)\|$, or

(ii) $f$ is weakly inward (i.e. $f(x) \in \overline{I_{\overline{P_{R}}}(x)}$, for each $x \in \overline{P_{R}}$ ), then $f$ has a fixed point in $\overline{P_{R}}$.

ProOF. By Theorem 1, there exists $u$ in $\overline{P_{R}}$ such that $\|u-f(u)\|=d\left(f(u), \overline{P_{R}}\right)$. Let $f$ satisfy (i). If $I_{\overline{P_{R}}}(u) \cap P=\overline{P_{R}}$, then $u=f(u)$; otherwise, there exists $y$ in $P \cap I_{\overline{P_{R}}}(u)=\overline{P_{R}}$ such that $\|y-f(u)\|<\|u-f(u)\|$, which contradicts our choice of $u$.

If $I_{\overline{P_{R}}}(u) \cap P \supsetneqq \overline{P_{R}}$ and assuming that $u \neq f(u)$, then there exists $y$ in $P \cap I_{\overline{P_{R}}}(u)$ such that $\|y-f(u)\|<\|u-f(u)\|$. With our choice of $u$, we know that $y \notin \overline{P_{R}}$. Therefore, there exists $z \in \overline{P_{R}}$ such that $y=u+c(z-u)$ for some $c>1$; that is,

$$
z=y / c+(1-1 / c) u=(1-\beta) y+\beta u,
$$

where $\beta=1-1 / c, 0<\beta<1$. Hence

$$
\begin{aligned}
\|z-f(u)\| & =\|(1-\beta) y+\beta u-f(u)\| \\
& \leq(1-\beta)\|y-f(u)\|+\beta\|u-f(u)\| \\
& <(1-\beta)\|u-f(u)\|+\beta\|u-f(u)\|=\|u-f(u)\|,
\end{aligned}
$$


which contradicts the choice of $u$. Therefore $u=f(u)$. It is clear to see that if $f$ satisfies (ii), then it also satisfies (i).

REMARK. Theorems 1 and 2 are proved by the author [10] for a closed ball in a Banach space. Here we generalize these results to cones. Note so far that we have not required $P \cap(-P)=\{0\}$.

Now we turn our attention to annulus in cones. We will prove that the above results are still true under suitable boundary conditions. We need the assumption that the norm $\|x\|$ is increasing with respect to $P$. Hence, from now on, we require that $P \cap(-P)=\{0\}$ in order to generate a natural order in $P$.

THEOREM 3. Let $f$ be a continuous $k$-set-contraction map $(0<k<1)$ from $\overline{P_{R}}$ into $P$, and let the norm $\|x\|$ be increasing with respect to $P$. If

$$
\|f(x)\| \geq\|x\| \text { for } x \in \partial P_{r}
$$

then there exists $u$ in $\overline{P_{r, R}}$ such that

$$
\|u-f(u)\|=d\left(f(u), \overline{P_{R}}\right)=d\left(f(u), \overline{P_{r, R}}\right) .
$$

Proof. Let $h$ be the same as in Theorem 1. Then $F(x) \equiv h(f(x)): \overline{P_{R}} \rightarrow \overline{P_{R}}$ is a continuous $k$-set-contraction map. We will show that $F$ has a nonzero fixed point in $\overline{P_{r, R}}$. From Li [9, Lemma 2], it suffices to show that

$$
\|F(x)\| \leq\|x\|, \quad \text { for } x \in \partial P_{R}, \quad \text { and } \quad\|F(x)\| \geq\|x\|, \quad \text { for } x \in \partial P_{r} .
$$

In fact, if $x \in \partial P_{R}$, then $x \in P$ and $\|x\|=R$. Since $F(x) \in \overline{P_{R}}$, we have $\|F(x)\| \leq R$, i.e. $\|F(x)\| \leq\|x\|$.

If $x \in \partial P_{r}$, then $x \in P$ and $\|x\|=r$. We consider the following cases:

(i) If $r \leq\|f(x)\| \leq R$, then $h(f(x))=f(x)$ by the definition of $h$. Therefore $\|F(x)\|=\|h(f(x))\|=\|f(x)\| \geq r=\|x\|$.

(ii) If $R<\|f(x)\|$, then $h(f(x))=R f(x) /\|f(x)\|$ and $\|F(x)\|=\|h(f(x))\|=$ $R>r=\|x\|$.

(iii) $\|f(x)\|<r$ is impossible, for, by assumption, we have $\|f(x)\| \geq\|x\|=r$ for $x \in \partial P_{r}$.

Therefore $\|F(x)\| \geq\|x\|$ for $x \in \partial P_{r}$. From Li [9, Lemma 2], there exists $u$ in $\overline{P_{r, R}}$ such that $F(u)=u$. By the same argument in proving Theorem 1 , we can prove that $\|u-f(u)\|=d\left(f(u), \overline{P_{R}}\right)=d\left(f(u), \overline{P_{r, R}}\right)$.

THEOREM 4. Let $f$ be the same as in Theorem 3. Moreover, if $f$ satisfies either one of the following conditions on the outer boundary $\partial P_{R}$ :

(i) for each $x \in \partial P_{R}$, with $x \neq f(x)$, there exists $y$ in $P \cap I_{\overline{P_{R}}}(x)$ such that $\|y-f(x)\|<\|x-f(x)\|$,

(ii) $f(x) \in \overline{I_{\bar{P}_{R}}(x)}$ for each $x \in \partial P_{R}$, then $f$ has a fixed point in $\overline{P_{r, R}}$.

ProOF. By Theorem 3, there exists $u$ in $\overline{P_{r, R}}$ such that $\|u-f(u)\|=d\left(f(u), \overline{P_{R}}\right)$. We will show that $u$ is a fixed point of $f$. If $u \in P_{R}$, then $\|u\|<R$ and there exists $\lambda, 0<\lambda<1$, such that $\lambda u+(1-\lambda) f(u) \in P_{R}$. Let $v=\lambda u+(1-\lambda) f(u)$. Then

$$
\|u-f(u)\|=d\left(f(u), \overline{P_{R}}\right) \leq\|v-f(u)\|=|\lambda|\|u-f(u)\| .
$$

Since $0<\lambda<1$, we must have $\|u-f(u)\|=0$, which implies that $u$ is a fixed point of $f$. If $u \in \partial P_{R}$ and $f$ satisfies (i), the same argument in proving Theorem 
2 shows that $u=f(u)$. Also we know that if $f$ satisfies (ii) then $f$ also satisfies (i).

COROLlaRY 1 (Li [9, LEMMA 2]). Let $f$ be the same as in Theorem 3. Moreover, if

$$
\|f(x)\| \leq\|x\| \quad \text { for } x \in \partial P_{R},
$$

then $f$ has a fixed point in $\overline{P_{r, R}}$.

Proof. $\|f(x)\| \leq R$ and $f(x) \in \overline{P_{R}}$ for $x \in \partial P_{R}$ since $\|f(x)\| \leq\|x\|$ for $x \in \partial P_{R}$. Since $\overline{P_{R}}$ is a subset of $I_{\overline{P_{R}}}(x)$, we have $f(x) \in I_{\overline{P_{R}}}(x)$. From Theorem 4, $f$ has a fixed point in $\overline{P_{r, R}}$.

REMARK. Li [9, Lemma 2] also proved that Corollary 1 is true for the boundary conditions

$$
\|f(x)\| \geq\|x\| \quad \text { for } x \in \partial P_{R}, \quad \text { and } \quad\|f(x)\| \leq\|x\| \quad \text { for } x \in \partial P_{r} .
$$

THEOREM 5. Let $f$ be a continuous condensing map from $\overline{P_{R}}$ into $P$, and let the norm $\|x\|$ be increasing with respect to $P$. If there is a $\delta>0$ such that

$$
\|f(x)\| \geq(1+\delta)\|x\| \quad \text { for } x \in \partial P_{r},
$$

then there exists $u$ in $\overline{P_{r, R}}$ such that

$$
\|u-f(u)\|=d\left(f(u), \overline{P_{R}}\right)=d\left(f(u), \overline{P_{r, R}}\right) .
$$

ProOF. Let $h$ be the same as in Theorem 1. Then $F(x) \equiv h(f(x)): \overline{P_{R}} \rightarrow \overline{P_{R}}$ is a continuous condensing map. We will show that $F$ has a nonzero fixed point in $\overline{P_{r, R}}$.

Take $\delta_{1}=\min \{\delta, R / r-1\}>0$. From Li [9, Theorem], it suffices to show that

$$
\|F(x)\| \leq\|x\| \quad \text { for } x \in \partial P_{R}, \quad \text { and } \quad\|F(x)\| \geq\left(1+\delta_{1}\right)\|x\| \quad \text { for } x \in \partial P_{r} .
$$

We note that Li's result is true for a continuous demicompact, 1-set-contraction map and we also note that a continuous condensing map is also demicompact and 1-set-contraction (e.g. $[\mathbf{1 5}$, p. 321]).

In fact, if $x \in \partial P_{R}$, then $x \in P$ and $\|x\|=R$. Since $F(x) \in \overline{P_{R}}$, we have $\|F(x)\| \leq R$, i.e. $\|F(x)\| \leq\|x\|$.

If $x \in \partial P_{r}$, then $x \in P$ and $\|x\|=r$. We consider the following cases:

(i) If $r \leq\|f(x)\| \leq R$, then $h(f(x))=f(x)$ by the definition of $h$. Therefore $\|F(x)\|=\|h(f(x))\|=\|f(x)\| \geq(1+\delta)\|x\| \geq\left(1+\delta_{1}\right)\|x\|$, by the assumption of $f$ on $\partial P_{r}$.

(ii) If $R<\|f(x)\|$, then $h(f(x))=R f(x) /\|f(x)\|$ and $\|F(x)\|=\|h(f(x))\|=R$. Since $R / r-1 \geq \delta_{1}$, we have $R \geq\left(1+\delta_{1}\right) r$. Therefore $\|F(x)\|=R \geq\left(1+\delta_{1}\right) r=$ $\left(1+\delta_{1}\right)\|x\|$.

(iii) $\|f(x)\|<r$ is impossible, for, by assumption, we have $\|f(x)\| \geq(1+\delta)\|x\|=$ $(1+\delta) r$.

Hence $\|F(x)\| \geq\left(1+\delta_{1}\right)\|x\|$ for $x \in \partial P_{r}$. From Li [9, Theorem], there exists $u$ in $\overline{P_{r, R}}$ such that $F(u)=u$. By the same argument in proving Theorem 1 , we can prove that

$$
\|u-f(u)\|=d\left(f(u), \overline{P_{R}}\right)=d\left(f(u), \overline{P_{r, R}}\right)
$$


THEOREM 6. Let $f$ be the same as in Theorem 5. Moreover, if $f$ satisfies either one of the following conditions on the outer boundary $\partial P_{R}$ :

(i) for each $x \in \partial P_{R}$, with $x \neq f(x)$, there exists $y$ in $P \cap I_{\overline{P_{R}}}(x)$ such that $\|y-f(x)\|<\|x-f(x)\|$, or

(ii) $f(x) \in \overline{I_{\overline{P_{R}}}(x)}$ for each $x \in \partial P_{R}$, then $f$ has a fixed point in $\overline{P_{r, R}}$.

PROOF. The proof is exactly the same as Theorem 4, except using Theorem 5 instead of Theorem 3.

Following the same argument as Corollary 1, we have the following

COROLlaRY 2. Let $f$ be the same as in Theorem 5. Moreover, if $\|f(x)\| \leq\|x\|$ for $x \in \partial P_{R}$, then $f$ has a fixed point in $\overline{P_{r, R}}$.

REMARK. $\mathrm{Li}$ [9, Theorem] proved that Corollary 2 is true for a continuous demicompact 1-set-contraction map $f$.

\section{REFERENCES}

1. H. Amann, Fixed point equations and nonlinear eigenvalue problems in ordered Banach spaces, SIAM Rev. 18 (1976), 620-709.

2. N. P. Các and J. A. Gatica, Fixed point theorems for mappings in ordered Banach spaces, J. Math. Anal. Appl. 71 (1979), 547-557.

3. K. Fan, Extensions of two fixed point theorems of F. E. Browder, Math. Z. 112 (1969), 234-240.

4. $\_$, Some properties of convex sets related to fixed point theorems, Math. Ann. 266 (1984), 519-537.

5. P. M. Fitzpatrick and W. V. Petryshyn, Fixed point theorems and the fixed point index for multivalued mappings in cones, J. London Math. Soc. (2) 12 (1975), 75-85.

6. M. Furi and A. Vignoli, On $\alpha$-nonexpansive mappings and fixed points, Atti. Accad. Naz. Lincei Rend. Cl. Sci. Fis. Mat. Natur. (8) 48 (1970), 195-198.

7. B. Halpern and G. M. Bergman, $A$ fixed point theorem for inward and outward maps, Trans. Amer. Math. Soc. 130 (1968), 353-358.

8. M. A. Krasonselsky, Positive solutions of operator equations, Noordhoff, Groningen, 1964.

9. G. Li, A new fixed point theorem on demi-compact 1-set-contraction mappings, Proc. Amer. Math. Soc. 97 (1986), 277-280.

10. T. C. Lin, A note on a theorem of Ky Fan, Canad. Math. Bull. 22 (1979), 513-515.

11. __ Convex sets, fixed points, variational and minimax inequalities, Bull. Austral. Math. Soc. 34 (1986), 107-117.

12. T. C. Lin and C. L. Yen, Applications of the proximity map to fixed point theorems in Hilbert space, J. Approx. Theory (to appear).

13. R. D. Nussbaum, The fixed point index for local condensing maps, Ann. Mat. Pura Appl. 89 (1971), 217-258.

14. __, Periodic solutions of some nonlinear autonomous functional differential equations, J. Differential Equations 14 (1973), 360-394.

15. W. V. Petryshyn, Structure of the fixed point sets of $k$-set-contractions, Arch. Rational Mech. Anal. 40 (1971), 312-328.

16. S. Reich, Approximate selections, best approximations, fixed points and invariant sets, J. Math. Anal. Appl. 62 (1978), 104-113.

17. B. N. Sadovski, A fixed point principle, Funct. Anal. Appl. 1 (1967), 151-153.

18. V. M. Sehgal and S. P. Singh, A theorem on the minimization of a condensing multifunction and fixed points, J. Math. Anal. Appl. 107 (1985), 96-102.

DEPARTMENT OF MATHEMATICAL SCIENCES, UNIVERSity OF WisCoNSin-MilWAUKEE, MILWAUKEe, WISCONSIN 53201 